\title{
BMJ Open Impact of antimicrobial stewardship managed by clinical pharmacists on antibiotic use and drug resistance in a Chinese hospital, 2010-2016: a retrospective observational study
}

\author{
Huaguang Wang, ${ }^{1}$ Han Wang, ${ }^{2}$ Xiaojia Yu, ${ }^{1}$ Hong Zhou, ${ }^{1}$ Boyu Li, ${ }^{1}$ Gang Chen, ${ }^{1}$ \\ Zhikang Ye, ${ }^{1}$ Ying Wang, ${ }^{1}$ Xiangli Cui, ${ }^{1}$ Yunying Zheng, ${ }^{1}$ Rui Zhao, ${ }^{1}$ Hui Yang, ${ }^{1}$ \\ Zihui Wang, ${ }^{1}$ Peng Wang, ${ }^{1}$ Chunxia Yang, ${ }^{1}$ Lihong Liu
}

To cite: Wang $\mathrm{H}$, Wang $\mathrm{H}, \mathrm{Yu} \mathrm{X}$, et al. Impact of antimicrobial stewardship managed by clinical pharmacists on antibiotic use and drug resistance in a Chinese hospital, 2010-2016: a retrospective observational study. BMJ Open 2019;9:e026072. doi:10.1136/ bmjopen-2018-026072

- Prepublication history and additional material for this paper are available online. To view these files, please visit the journal online (http://dx.doi org/10.1136/bmjopen-2018026072).

Received 21 August 2018 Revised 29 May 2019 Accepted 10 June 2019
Check for updates

(c) Author(s) (or their employer(s)) 2019. Re-use permitted under CC BY-NC. No commercial re-use. See rights and permissions. Published by BMJ.

${ }^{1}$ Beijing Chaoyang Hospital, Capital University of Medical Sciences, Beijing, China ${ }^{2}$ Department of Maternal and Child Health, School of Public Health, Peking University, Beijing, China

Correspondence to Lihong Liu; liulihong@bjcyh.com

\section{ABSTRACT}

Objectives 'National Special Stewardship in the Clinical Use of Antibiotics' was put forward in July 2011 in China. We aimed to retrospectively evaluate the impact of antimicrobial stewardship (AMS) managed by clinical pharmacists on antibiotic utilisation, prophylaxis and antimicrobial resistance (AMR).

Design This was a retrospective observational study of trends in antibiotic use and AMR in the context of AMS.

Setting Beijing Chaoyang Hospital, a 1400-bed tertiary hospital, in China.

Data and participants Antibiotic prescriptions from 820 doctors included all outpatients $(n=17766$ 637) and inpatients $(n=376627)$ during 2010-2016. Bacterial resistance data were from all inpatients $(n=350699)$ during 2011-2016.

Interventions Multiaspect intervention measures were implemented by clinical pharmacists (13 persons), for example, formulating the activity programme and performance management, advising on antibacterial prescriptions and training.

Outcome measures The proportion of antibiotic prescriptions among outpatients and inpatients, intensity of consumption in defined daily dose (DDD)/100 bed-days, antibiotic prophylaxis in type I incision operations and resistance rates of Escherichia coli, Klebsiella pneumoniae and Pseudomonas aeruginosa were retrospectively analysed.

Results The proportion of antibiotic prescriptions decreased in outpatients (from 19.38\% to 13.21\%) and in inpatients (from $64.34 \%$ to $34.65 \%$ ), the intensity of consumption dropped from 102.46 to $37.38 \mathrm{DDD} / 100$ beddays. The proportion of antibiotic prophylaxis decreased from $98.94 \%$ to $18.93 \%$. The proportion of rational timing of initial dose increased from $71.11 \%$ to $96.74 \%$, the proportion of rational duration rose from $2.84 \%$ to $42.63 \%$. Time series analysis demonstrated the resistance rates of $E$. coli and $P$. aeruginosa to fluoroquinolones decreased, the incidence rate of methicillin-resistant Staphylococcus aureus also decreased, whereas the resistance rates of $E$. coli and K. pneumoniae to carbapenems increased. The antibiotic use was partly positively correlated with AMR.
Strengths and limitations of this study

Our study described the entire process of antimicrobial stewardship, from management of antibiotic use to antimicrobial resistance (AMR) monitoring.

- Time series analysis, a better tool, was applied to analyse the change trends in antibiotic utilisation and AMR.

- By exploring the correlation between antibiotic use and AMR, this study may indicate some potential directions for controlling the prevalence of carbapenem-resistant Enterobacteriaceae and methicillin-resistant Staphylococcus aureus.

- This was a retrospective observational study without simultaneous control group, the bias could not be well controlled; the evaluation of prophylactic antibiotic use by different clinical pharmacists might have individual differences.

Conclusions AMS had an important role in reducing antibiotic use and surgical antibiotic prophylaxis. The AMR was positively correlated with antibiotic consumption to some extent.

\section{INTRODUCTION}

In 2004, the first Guidelines for the Clinical Use of Antibiotics (Guidelines for short) was issued by the National Health and Family Planning Commission (NHFPC, originally called the Ministry of Health) of the People's Republic of China, describing the characteristics of all types of antibiotics and appropriate use in treatment and prevention of infectious diseases; the Guidelines were updated in 2015. Regretfully, not all medical staff knew about the Guidelines or their significance. Today, antimicrobial resistance (AMR) is one of the greatest threats to global health. There are four main factors contributing to 
the spread of AMR: inappropriate use of antibiotics in the community and in hospitals, misuse of antibiotics in animal production and agriculture and the presence of resistant bacteria in the environment. The former three factors could aggravate the last one. ${ }^{1}$ Chinese data from the Ministry of Health National Antibacterial Resistance Surveillance Net showed that AMR has been rising steadily each year. ${ }^{2}$

In 2001, WHO began to take measures to combat the spread of AMR and strongly recommended governments to implement antimicrobial stewardship (AMS). ${ }^{3}$ On World Health Day 2011, AMR was also selected as the theme. In response to AMR, in 2011 the NHFPC of China put forward 'National Special Stewardship in the Clinical Use of Antibiotics', ${ }^{4}$ the historically strictest management of antibiotics up to that date. The NHFPC set many goals for the clinical use of antibiotics, including restriction of antibiotic use in outpatients and inpatients and restriction of antibiotic prophylactic use in clean operations, to promote rational antibiotic use and control AMR. These goals are described in detail below. This special stewardship policy mainly covered secondary and tertiary public hospitals and took effect on 1 July 2011. After that date, these hospitals were required to report data of antibiotic use to the government every month.

In recent years, some studies have reported that AMS had positive effects on controlling antibiotic-resistant pathogens, rational use of antibiotics and cost savings, ${ }^{56}$ highlighting the importance of AMS. There were also some studies $^{7-11}$ that analysed the correlation between antibiotic use and AMR, although these all demonstrated the effectiveness of AMS, but the studied population, antibiotic and pathogen are different, and the results of correlation between antibiotic use and AMR were not exactly the same.

The aim of this study was to evaluate the impact of AMS on antibiotic use and AMR trends, to share our successful management experience and to identify existing problems. In addition, because the doctors' prescription behaviours and antibiotic variety are different in each country or region, so is the status of AMR, therefore we sought to demonstrate the correlation between antibiotic use and antimicrobial resistance rate of common nosocomial pathogens, using data from all inpatients in our hospital.

\section{METHODS}

\section{Study design}

According to the requirements of the national policy, 'Special Stewardship in the Clinical Use of Antibiotics' was a 3-year plan (2011-2013). In April 2014, the NHFPC issued a notice regarding implementing stewardship of antibacterial use in the $\operatorname{clinic}^{12}$; its aim was to continuously maintain the positive effects gained during the previous 3 years. Accordingly, in our retrospective study, phases were divided into three stages, as follows. Stage 1: baseline phase (July 2010 to June 2011); stage 2: intervention phase (July 2011 to December 2013); and stage 3: stability phase (January 2014 to December 2016).

\section{Patient and public involvement}

The antibiotic utilisation data were extracted directly from the hospital information system (HIS) and electronic medical records of all patients (2010-2016). The patient's personal information was hidden. The bacterial resistance data from all inpatients (2011-2016) were provided by the Department of Infectious Diseases and Clinical Microbiology. Clinical sample sources included blood, cerebrospinal fluid, pleural effusion, ascites, urine and sputum, etc. Duplicate isolates, defined as the isolates of the same species that showed the same susceptibility results at the same site for each patient in different days, were excluded, only the first isolated strain was included in the study (excluding isolates of surveillance cultures).

\section{Ethics statement}

Because the patient's privacy was not violated in the study, so the Ethics Committee agreed exemption applications of informed consent.

\section{Multiaspect intervention measures Organisation construction}

To implement the programme 'National Special Stewardship in the Clinical Use of Antibiotics', an AMS group was set up in our hospital, which was attached to the Drug and Therapeutics Committee (DTC). The AMS group was composed of administrators, clinicians, infectious disease physicians, pharmacists, microbiologists and information staff, and included a leadership group and expert group. The leadership group was responsible for work deployment and supervision, whereas the expert group was responsible for technical guidance, participation in consultations, training doctors on rational use of antibiotics and implementation of AMS monitoring (such as data collection and report, prescription review and feedback, AMR monitoring, etc). Generally, the medical department led AMS in many hospitals in China, but in our hospital, the pharmacy department was the leading department, for the following reasons: (1) the pharmacy department in our hospital is a technical and functional section. The pharmacy director is responsible for medication use; (2) there are many clinical pharmacists, such as infectious disease pharmacists who have sufficient knowledge and clinical experience to manage AMS; (3) clinical pharmacists work in the clinical departments every day, so they could give their professional advice regarding antibiotic use directly to doctors.

\section{Formulating the activity programme and administrative}

\section{intervention}

The AMS group formulated the activity programme of stewardship and some regulations on antibiotic use were issued, as follows: (1) antibiotic classification management system. All antibiotics were classified as non-restricted, restricted and special grade antibiotics. Physicians with different professional titles were matched 
Table 1 Goals of clinical antibiotic use established by the NHFPC in 2011

\begin{tabular}{ll}
\hline Antibiotic outcome measures & Goals \\
\hline $\begin{array}{l}\text { 1. Proportion of inpatients receiving } \\
\text { antibiotics }\end{array}$ & $\leq 60 \%$ \\
$\begin{array}{l}\text { 2. Proportion of outpatients receiving } \\
\text { antibiotics }\end{array}$ & $\leq 20 \%$ \\
$\begin{array}{l}\text { 3. Intensity of inpatients' antibiotic } \\
\text { consumption }\end{array}$ & $\begin{array}{l}\leq 40 \text { DDD/100 bed- } \\
\text { days }\end{array}$ \\
$\begin{array}{l}\text { 4. Proportion of antibiotic prophylaxis } \\
\text { in patients receiving type I incision } \\
\text { operations/clean operations }\end{array}$ & $\leq 30 \%$ \\
$\begin{array}{l}\text { 5. Timing of initial dose of preoperative } \\
\text { antibiotic prophylaxis }\end{array}$ & $\begin{array}{l}\text { Within 0.5-2 hours } \\
\text { before surgical } \\
\text { incision }\end{array}$ \\
$\begin{array}{l}\text { 6. Duration of antibiotic prophylaxis } \\
\text { in patients receiving type I incision } \\
\text { operations/clean operations }\end{array}$ & $\begin{array}{l}\text { Within } 24 \text { hours } \\
\text { after the end of } \\
\text { operation }\end{array}$ \\
\hline
\end{tabular}

DDD, defined daily dose; NHFPC, National Health and Family Planning Commission.

to the corresponding grade of antibiotic prescribing privileges; (2) management system of antibiotic prescribing privileges. In May 2012, the Regulations on Clinical Applications of Antibiotics were issued by the NHFPC, which took effect on 1 August 2012. These were the first valid regulations on antibiotics in China. ${ }^{13}$ The regulations required that physicians would not be given antibiotic prescribing privileges until they passed an exam, after completing training on rational use of antibiotics. This prescribing privilege restriction was embedded into the HIS; (3) regulation of perioperative prophylactic antibiotic use in clean operations, in which the choice of antibiotics, dose, timing of the initial dose and duration of antibiotic prophylaxis were described.

According to the requirements of the national antibiotic stewardship programme, ${ }^{4}$ the AMS group established the goals for antibiotic application in the hospital (table 1).

\section{Performance management}

Every year, the directors of clinical departments were asked by the director of the hospital to sign responsibility agreements for antibiotic use. Hospital leaders and the pharmacy director, together with clinical pharmacists, established or updated the performance appraisal system for antibiotic use, which indicated the circumstances to be rewarded or penalised. For example, if clinical departments did not accomplish their goals, the directors would be fined $¥ 1000-3000$, and doctors would be fined $¥ 300-500$. If the clinical departments accomplished their goals, the directors would be rewarded with $¥ 1000-5000$ and doctors with $¥ 300-1000$, which were greater than the amounts of fines.

\section{Antibiotic prescription evaluation and training}

Retrospective rationality evaluation of antibiotic prescriptions for outpatients, emergency room patients and inpatients was performed monthly by clinical pharmacists. For example, some doctors used moxifloxacin to treat urinary tract infections, which did not conform to the recommendation of guideline and medicine specification; the combination of imipenem/cilastatin and metronidazole was unsuitable, the latter was unnecessary. Clinical pharmacists would contact the doctors to modify the prescriptions. Inappropriate prescriptions would be flagged in the Antibacterial Monitoring Report published by the pharmacy department each month; this report was made available to all medical staff. According to the frequency and severity of inappropriate prescriptions, some doctors would be fined.

Clinical pharmacists were responsible for training the medical staff on rational use of antibiotics. Training was conducted every 6 months in two forms: (1) clinical pharmacists gave lessons to the medical staff in the lecture hall, they need to complete an exam after class; (2) clinical pharmacists and the medical department jointly made online learning and exam, medical staff was required to finish it. If necessary, pharmacists would go to the clinical departments to give lectures.

\section{Multiple cooperation}

Antibiotics data monitoring could not be implemented without the support of the information department. At the start of AMS at our hospital, data extraction modules were embedded into the HIS after discussions between clinical pharmacists and information personnel. Later, an automatic prescription screening system was also included in the HIS, which could intercept inappropriate prescriptions, such as repeated use or unreasonable combinations. Furthermore, clinical pharmacists took part in the Core Expert Meeting of Antibacterial Application held by the Infection Management Office, to discuss usage problems with carbapenems and glycopeptides. If inappropriate use was confirmed by the experts, the relevant physician would be penalised $¥ 100-200$ fine.

\section{Data collection and outcome measures}

Antibiotic outcome measures are shown in table 1. The antibiotic utilisation data were collected directly from the HIS. Antibiotic consumption was standardised according to the Anatomical Therapeutic Chemical (ATC) classification system and the DDD was used as a measuring unit, as recommended by the WHO Collaborating Center for Drug Statistics Methodology. ${ }^{14}$ The intensity of inpatients' antibiotic consumption was expressed as DDD/100 bed-days. Information regarding type I incision operations was extracted from inpatients' electronic medical records. The outcome measures of AMR included the resistance rates of Escherichia coli, Klebsiella pneumoniae and Pseudomonas aeruginos $a$ and incidence rate of methicillin-resistant Staphylococcus aureus (MRSA) in our hospital. The bacteriological data were obtained from the clinical microbiology laboratory. We 
analysed the correlation between antibiotic consumption and AMR.

\section{Statistical analysis}

Segmented regression analysis of interrupted time series was used to analyse the monthly data of antibiotic utilisation, which were divided into three stages (the baseline phase, intervention phase and stability phase), to illustrate the effect of AMS. The statistical model in this study was as follows ${ }^{15}$ :

$$
\begin{aligned}
Y_{t} & =\beta_{0}+\beta_{1} \times \text { time }_{t}+\beta_{2} \times \text { intervention }_{t}+\beta_{3} \times \text { time after } \\
& \text { intervention }_{t}+\beta_{4} \times \text { stability }_{t}+\beta_{5} \times \text { time after stability }_{t}+e_{t}
\end{aligned}
$$

In this model, $Y_{t}$ is the average monthly value of the outcome measure at month $t ; \beta_{0}$ estimates the level change in the outcome during the baseline phase; $\beta_{1}$ estimates the trend change during the baseline phase; $\beta_{2}$ estimates the level change during the intervention phase; $\beta_{3}$ estimates the trend change during the intervention phase; $\beta_{4}$ estimates the level change during the stability phase and $\beta_{5}$ estimates the trend change during the stability phase. The parameter level was the value of a time series at the beginning of a given time series; the parameter trend was the rate of change in an outcome measure; time was a continuous variable indicating time in months at time $t$ starting from the baseline phase (time 0 ); intervention was an indicator for time $t$ occurring before (intervention $=0$ ) or after (intervention $=1$ ) the multiaspect intervention, which started at month 13 (July 2011); time after intervention was a continuous variable counting the months after the intervention; stability was an indicator for time $t$ occurring before (stability $=0$ ) or after stability (stability $=1$ ), which started at month 43 (January 2014); time after stability was a continuous variable counting the months after stability. The error term, $e_{t}$, represented variation unexplained by the segmented regression model.

Comparisons of the average monthly values of outcome measures for antibiotic use during the three phases were conducted using the Bonferroni test. Box charts were plotted for data visualisation, with error bars representing SD.

In addition, a time series analysis model (autoregressive integrated moving average $)^{8}$ was used to analyse the trends in annual antibiotic use, AMR trends and incidence trend of MRSA from 2011 to 2016. The $\beta$ value indicated the variation of dependent variables when independent variables changed one unit at uniform time intervals. Pearson's correlation coefficients were used to examine the relationships between antimicrobial resistance rate, the incidence rate of MRSA and antibiotic use.

All statistics were performed using SAS, V.9.3 (SAS Institute, Cary, North Carolina, USA). All reported $p$ values were two-sided, with $\mathrm{p}<0.05$ considered to be statistically significant.

\section{RESULTS}

\section{Change trends in antibiotic utilisation rate and intensity}

Changes in the proportion of antibiotic prescriptions in outpatients and inpatients during the baseline, intervention and stability phases are shown in figure $1 \mathrm{~A}, \mathrm{~B}$,
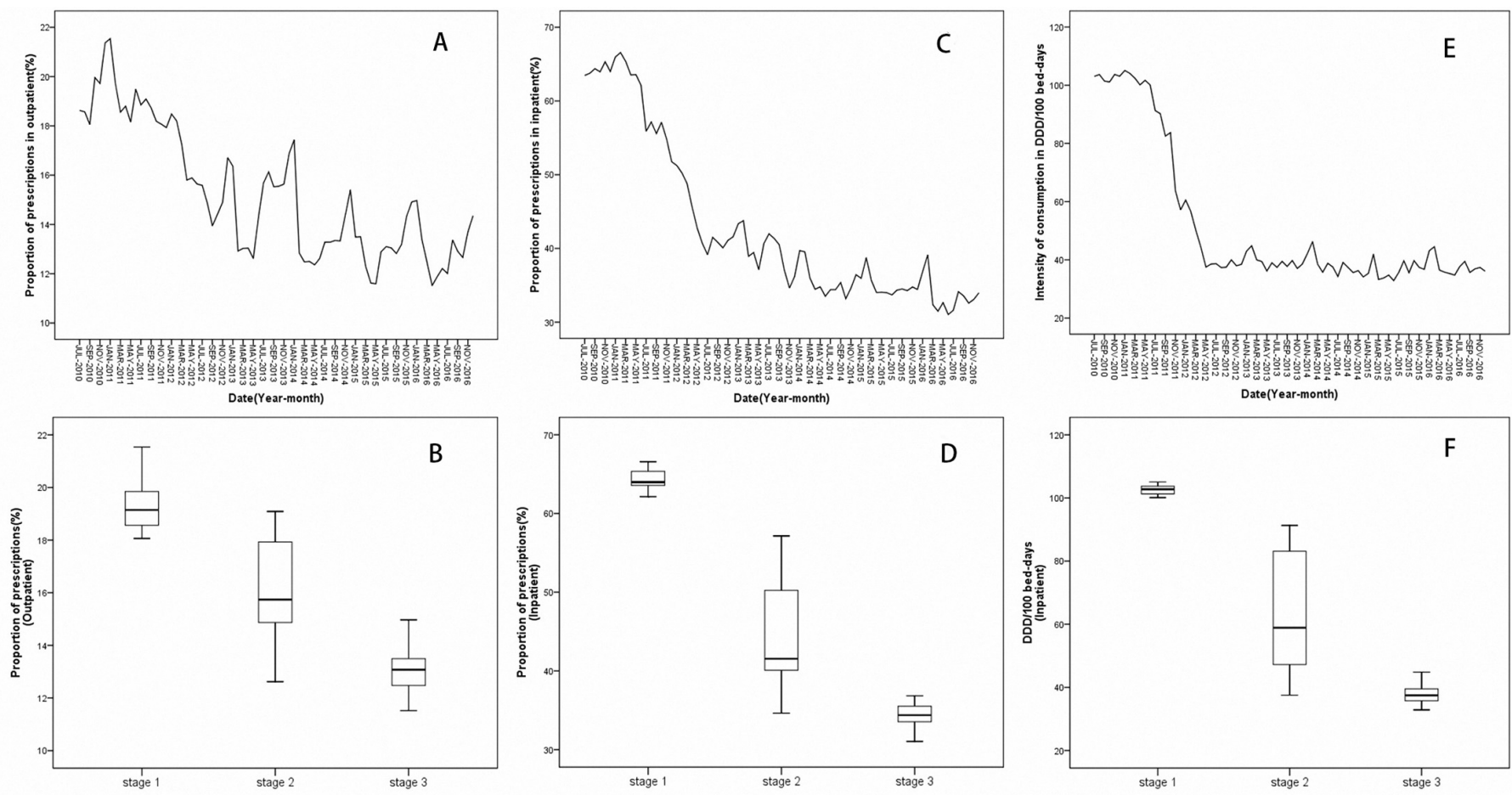

Figure 1 Changes in proportion of antibiotic prescriptions and intensity of consumption. Time series curves of each monthly value of antibiotic prescribing proportions, plotted for outpatients $(A)$ and inpatients $(C)$. Each monthly value of intensity of antibiotic consumption was plotted for inpatients $(E)$. The Bonferroni test was conducted to compare these data in three stages, for antibiotic prescribing proportion among outpatients (B) and inpatients (D), and intensity of antibiotic consumption (F). Stages 1,2 and 3 on the $x$-axis represent the baseline phase, intervention phase and stability phase, respectively. 
Table 2 Time series analysis of change trends in antibiotic utilisation

\begin{tabular}{llllll}
\hline Antibiotic outcome measures & $\begin{array}{l}\boldsymbol{\beta}_{1} \text { trend } \\
\text { (baseline) }\end{array}$ & $\begin{array}{l}\boldsymbol{\beta}_{2} \text { level } \\
\text { (intervention) }\end{array}$ & $\begin{array}{l}\boldsymbol{\beta}_{3} \text { trend } \\
\text { (intervention) }\end{array}$ & $\begin{array}{l}\boldsymbol{\beta}_{4} \text { level } \\
\text { (stability) }\end{array}$ & $\begin{array}{l}\boldsymbol{\beta}_{5} \text { trend } \\
\text { (stability) }\end{array}$ \\
\hline Proportion-O & $-0.01(0.04)$ & $0.55(0.83)$ & $-0.33(0.12)^{\star}$ & $0.48(0.85)$ & $-0.19(0.14)$ \\
Proportion-I & $-0.25(0.10)^{\star}$ & $-5.03(2.22)^{\star}$ & $-0.59(0.29)^{\star}$ & $3.61(2.23)$ & $-0.66(0.74)$ \\
\hline Intensity-I & $-0.04(0.04)$ & $-7.44(3.62)^{\star}$ & $-6.46(0.56)^{\star \star \star}$ & $4.20(1.45)^{\star \star}$ & $0.70(0.19)^{\star \star \star}$ \\
Proportion-type I & $-0.10(0.04)^{\star}$ & $-7.26(2.92)^{\star}$ & $-5.71(0.61)^{\star \star \star}$ & $-0.18(1.44)$ & $-0.12(0.12)$ \\
\hline Timing-type I & $-0.01(0.07)$ & $0.64(1.72)$ & $1.18(0.59)^{\star}$ & $1.63(2.00)$ & $-0.17(0.24)$ \\
\hline Duration-type I & $0.28(0.06)^{\star \star \star}$ & $8.78(2.15)^{\star *}$ & $0.10(0.27)$ & $5.35(1.44)^{\star \star \star}$ & $-1.19(0.19)^{\star \star \star}$ \\
\hline
\end{tabular}

Outcomes of antibiotic utilisation included proportion of antibiotic prescriptions in outpatients (Proportion-O), inpatients (Proportion-I) and intensity of consumption in inpatients (Intensity-I). Outcomes of antibiotic prophylaxis included proportion of prophylaxis (Proportion-type I), proportion of rational timing (Timing-type I) and proportion of rational duration (Duration-type I). Parameters of $\beta 1-\beta 5$ were expressed as mean (SE), which represented the changes in level and trend.

${ }^{\star} \mathrm{P}<0.05 ;{ }^{* *} \mathrm{p}<0.01 ;{ }^{* \star} \mathrm{p}<0.001$.

$\mathrm{C}$ and $\mathrm{D}$. The associated parameters of time series analysis are summarised in table 2 . The proportion of antibiotic prescriptions in outpatients and inpatients declined by $0.33 \%(p<0.05)$ and by $0.59 \%(p<0.05)$ each month during the intervention stage, respectively. Bonferroni tests (figure 1B) showed that the proportion of antibiotic prescriptions in outpatients was reduced from 19.38\% during the baseline phase to $13.21 \%$ during the stability phase $(\mathrm{p}<0.05)$. The proportion of antibiotic prescriptions among inpatients decreased significantly from $64.34 \%$ during the baseline phase to $34.65 \%$ during the stability phase $(\mathrm{p}<0.05)$ (figure $1 \mathrm{D})$. Figure $1 \mathrm{E}$ and table 2 show that the intensity of inpatients' antibiotic consumption decreased significantly by $6.46 \mathrm{DDD} / 100$ bed-days $(p<0.001)$ per month during the first year of the intervention stage. Figure 1F shows the intensity of consumption dropped from the baseline phase to the stability phase (102.46 vs $37.38 \mathrm{DDD} / 100$ bed-days; $\mathrm{p}<0.05)$. All the outcomes mentioned above met the national standards. In the stability phase, the $\beta_{5}$ value for the intensity of consumption $(0.70 ; \mathrm{p}<0.001)$ implied a gradually increasing trend; this still met national standards.

\section{Change trends of antibiotic prophylaxis in type I incision operations}

The proportion of antibiotic prophylaxis in patients undergoing type I incision operations was significantly reduced by $5.71 \%(\mathrm{p}<0.001)$ monthly during the first year of the intervention phase (figure $2 \mathrm{~A}$, table 2), decreasing from $98.94 \%$ during the baseline phase to $18.93 \%$ during the stability phase $(\mathrm{p}<0.05)$ (figure $2 \mathrm{~B})$. The proportion of rational timing of the initial dose increased by $1.18 \%$ $(\mathrm{p}<0.05)$ each month during the intervention stage (figure 2C, table 2), also increasing from $71.11 \%$ during the baseline phase to $96.74 \%$ during the stability phase $(p<0.05)$ (figure 2D). These two outcomes all eventually reached national standards. Although the proportion of rational duration of antibiotic prophylaxis showed an increasing trend during the intervention phase $(0.10$; $\mathrm{p}<0.05)$, the difference was not statistically significant (figure 2E, table 2). However, in the stability phase, this showed a decreasing trend $(-1.19 ; \mathrm{p}<0.001)$, which did not meet the national standard $(\geq 90 \%)$. Figure $2 \mathrm{~F}$ shows the proportion of rational duration, increasing from $2.84 \%$ during the baseline phase to $42.63 \%$ during the stability phase $(\mathrm{p}<0.05)$.

Trends in resistance rates for common Gram-negative bacilli and incidence rate of MRSA, 2011-2016

Time series analysis demonstrated a significant increase in the resistance rates of $E$. coli to carbapenems during 2011-2016 $(\mathrm{p}<0.05)$. The $\beta$ value indicated that the resistance rates of $E$. coli to imipenem and meropenem increased by $0.27 \%$ and $0.22 \%$ each year, respectively. However, the resistance rates of $E$. coli to levofloxacin and ciprofloxacin significantly decreased by $1.62 \%$ and $1.40 \%$ each year, respectively $(\mathrm{p}<0.01$ and $\mathrm{p}<0.001)$ (table 3$)$.

Time series analysis demonstrated a significant increase in the resistance rates of $K$. pneumoniae to carbapenems $(\mathrm{p}<0.05)$. The $\beta$ value indicated that the resistance rates of $K$. pneumoniae to imipenem and meropenem increased by $1.29 \%$ and $1.14 \%$ each year, respectively. The resistance rates of $K$. pneumoniae to fluoroquinolones (FQs) remained stable (table 4 ).

Time series analysis showed a significant decrease in the resistance rates of $P$. aeruginosa to FQs $(\mathrm{p}<0.05$ and $\mathrm{p}<0.01)$. The $\beta$ value indicated that the resistance rate of $P$. aeruginos a to levofloxacin and ciprofloxacin decreased by $4.78 \%$ and $2.27 \%$ each year, respectively. Resistance rates of $P$. aeruginosa to carbapenems remained stable (table 5).

Our study showed that the incidence rate of nosocomial MRSA decreased significantly by $5.26 \%$ each year, declining from $68.0 \%$ (2011) to $37.5 \%$ (2016) $(\mathrm{p}<0.001)$ (online supplementary table S1).

\section{Correlation between antibiotic consumption and AMR}

Because carbapenems and FQs are often used for nosocomial infection, we focused on evaluating the impact of use of these drugs on AMR. We found that the intensity of consumption of imipenem/cilastatin significantly increased from 0.59 to $1.36 \mathrm{DDD} / 100$ bed-days $(\mathrm{p}<0.01)$. 

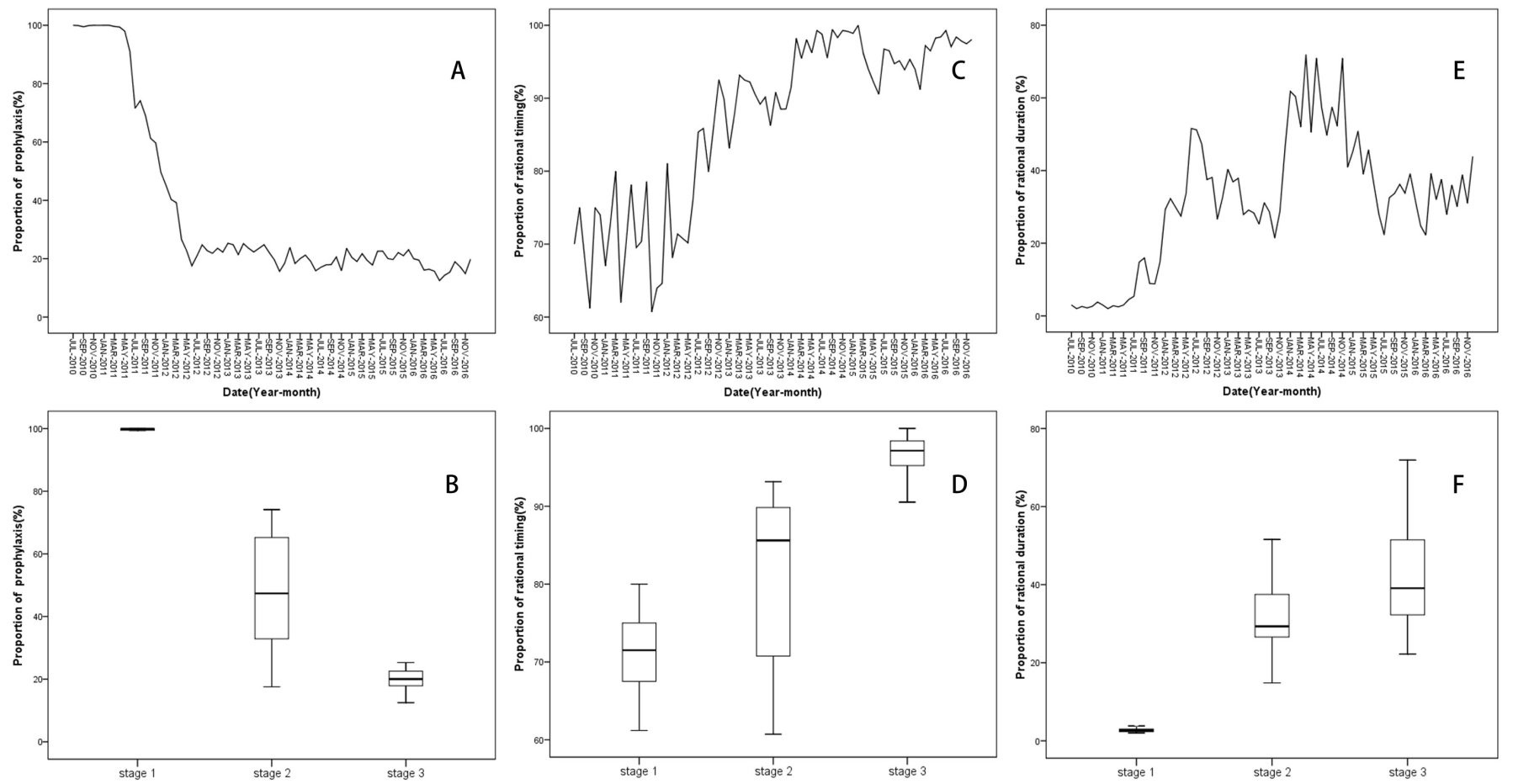

Figure 2 Changes in antibiotic prophylaxis in type I incision operations. Time series curves of each monthly value of antibiotic prophylaxis were plotted for proportion of prophylaxis $(A)$, proportion of rational timing $(C)$ and proportion of rational duration (E). These data in three stages were compared by Bonferroni test (B, D and F). Stages 1, 2 and 3 on the $\mathrm{x}$-axis represent the baseline phase, intervention phase and stability phase, respectively.

However, the intensity of consumption of FQs significantly decreased each year $(\mathrm{p}<0.01$ and $\mathrm{p}<0.05)$, respectively (online supplementary table S2).

Increased consumption of imipenem/cilastatin was correlated with the prevalence of imipenem-resistant $E$. coli $(\mathrm{r}=0.8651, \mathrm{p}<0.05)$. Similarly, decreased consumption of FQs was associated with the decreased resistance rate of $E$. coli to levofloxacin and ciprofloxacin ( $\mathrm{r}=0.8954 \mathrm{and}$ $\mathrm{r}=0.8950$, respectively; $\mathrm{p}<0.05$ ) (table 6 ).

There was a relationship between the increased resistance rate of $K$. pneumoniae to imipenem/cilastatin and increased intensity of consumption of imipenem/cilastatin $(\mathrm{r}=0.9050, \mathrm{p}<0.05)$. Although time series analysis showed a stable trend in the resistance rate of $K$. pneumoniae to ciprofloxacin (table 4), there was still a significantly positive correlation between the prevalence of ciprofloxacin-resistant $K$. pneumoniae and use of ciprofloxacin $(\mathrm{r}=0.9209, \mathrm{p}<0.01) \quad($ table 6$)$.
Table 6 indicates that the resistance rate of $P$. aeruginosa to FQs was correlated with the consumption of FQs $(r=0.8954, p<0.05$ for levofloxacin and $r=0.9282$, $\mathrm{p}<0.01$ for ciprofloxacin).

The incidence rate of MRSA was positively correlated with the consumption of FQs $(r=0.9450, p<0.01$ for levofloxacin and $\mathrm{r}=0.8883, \mathrm{p}<0.05$ for ciprofloxacin). However, we found that the incidence rate of MRSA was negatively correlated with the consumption of imipenem/cilastatin $(\mathrm{r}=-0.9611, \mathrm{p}<0.01)($ table 6$)$.

\section{DISCUSSION}

The global mortality attributable to AMR is estimated to reach 10 million annually by 2050 , which would make it one of the leading causes of death, with an economic impact of up to US $\$ 100$ trillion. ${ }^{16}$ Therefore, many countries worldwide have implemented AMS, with many

Table 3 Trend changes in antimicrobial resistance of Escherichia coli to carbapenems and fluoroquinolones from 2011 to 2016

\begin{tabular}{|c|c|c|c|c|c|c|c|c|c|}
\hline \multirow[b]{2}{*}{ Antibiotic } & \multicolumn{6}{|c|}{ Resistance rate (\%), by year } & \multicolumn{3}{|c|}{ Time series analysis } \\
\hline & 2011 & 2012 & 2013 & 2014 & 2015 & 2016 & Trend & $\beta$ & $P$ value \\
\hline Imipenem & 0 & 0 & 0.1 & 0.4 & 0.5 & 1.5 & Increasing & 0.2657 & 0.0239 \\
\hline Levofloxacin & 61.3 & 61.3 & 59.1 & 57.7 & 55.5 & 53.9 & Decreasing & -1.6191 & 0.0013 \\
\hline Ciprofloxacin & 64.3 & 64.3 & 61.2 & 61.4 & 58.7 & 58.2 & Decreasing & -1.4038 & 0.0002 \\
\hline
\end{tabular}


Table 4 Trend changes in antimicrobial resistance of Klebsiella pneumoniae to carbapenems and fluoroquinolones from 2011 to 2016

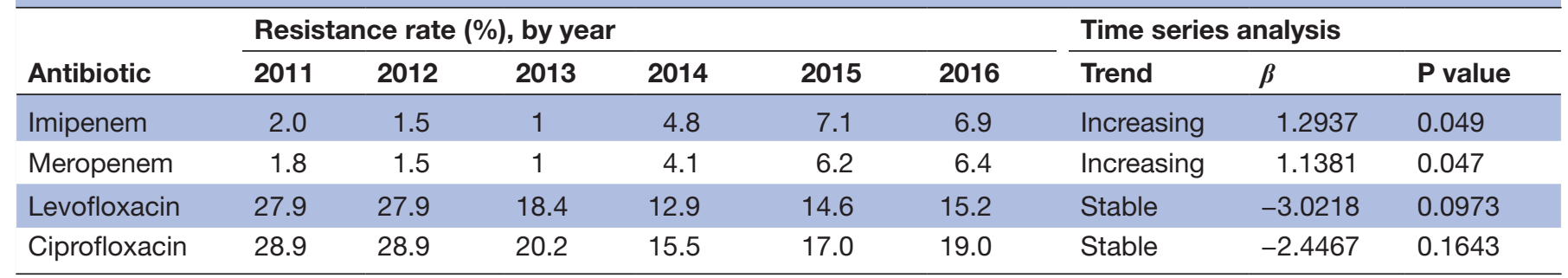

positive effects in the rational use of antibiotics and healthcare cost savings. ${ }^{17-21}$

The implementation of AMS in our hospital is managed by clinical pharmacists and supported by the DTC, while multiple sectors participate in it. AMS includes a multifaceted approach to combat the spread of AMR. Except the regular management strategy (such as multidisciplinary consultation, nosocomial infection control, prescription prospective audit, prescription evaluation and feedback, publicity and education, etc), our hospital established the reward and punishment mechanism aiming to arouse the doctor's attention to the rational use of antibiotics, which is slightly different from the existing intervention model and is unique among the published studies of AMS, but it reflects the current situation of some Chinese hospitals. After many years of AMS, the proportion of antibiotic prescriptions decreased to $13.21 \%$ among outpatients and $34.65 \%$ among inpatients. The intensity of antibiotic consumption was reduced to 37.38 DDD/100 bed-days. These outcomes are similar to the study by Bao et $a l .{ }^{15}$ Regarding antibiotic prophylaxis in type I incision operations, the proportion of antibiotic prophylaxis decreased to $18.93 \%$ and the proportion of rational timing of the initial dose increased to $96.74 \%$. Only the proportion of rational duration of antibiotic prophylaxis $(42.63 \%)$ did not reach the national standard, for the following reasons. First, coronary artery bypass graft (CABG) surgery belongs to type I incision operations. According to the Clinical Practice Guidelines for Antimicrobial Prophylaxis in Surgery, ${ }^{22}$ the duration of prophylaxis for cardiothoracic procedures is up to 48 hours, with no supporting evidence. Chinese Guidelines suggest prophylactic duration be no more than 48 hours. But CABG involves important viscera, in which the consequences of infection would be severe. Therefore, doctors hesitate to stop antibiotics within 48 hours after surgery. Second, in orthopaedic surgeries, such as open reduction and plate or screw internal fixation of fractures, in consideration of implants doctors also hesitate to stop antibiotics within 24 hours after surgery. Third, the difficult doctor-patient relationships in China make physicians hesitant about premature discontinuation of antibiotics.

The aim of AMS is to limit the prevalence of AMR. The results showed that with decreased intensity of FQ consumption, the resistance rates of E. coli and P. aeruginosa to FQs and incidence rate of MRSA showed decreasing trends, and they were positively correlated. This implied that controlling the use of FQs might limit the prevalence of AMR as well as limit the emergence of MRSA; the latter is consistent with previous studies. $^{2324}$ Other studies ${ }^{25-28}$ have reported that a reduction in second-generation/third-generation cephalosporins and clindamycin contributed to a reduction in both incidence rate of MRSA and prevalence density of MRSA bacteraemia. In our study, we also found that the incidence rate of MRSA was negatively correlated with imipenem/cilastatin use, which was difficult to explain. To our knowledge, few studies have obtained results similar to ours. Lai et al reported ${ }^{29}$ a significant correlation between increased use of linezolid and teicoplanin and decreased prevalence of MRSA. Therefore, we theorise that the reduced use of non-special grade antibiotics (such as FQs and others) leads to a compensatory increased use of carbapenems; however, this negative correlation requires further exploration. In addition, we found the resistance rate of $E$. coli and $K$. pneumoniae to carbapenems showed an increasing trend, meaning that carbapenem-resistant Enterobacteriaceae (CRE) could pose a serious threat. On 2 March 2017, the NHFPC issued a notice regarding further reinforcement in management

Table 5 Trend changes in antimicrobial resistance of Pseudomonas aeruginosa to carbapenems and fluoroquinolones from 2011 to 2016

\begin{tabular}{|c|c|c|c|c|c|c|c|c|c|}
\hline \multirow[b]{2}{*}{ Antibiotic } & \multicolumn{6}{|c|}{ Resistance rate (\%), by year } & \multicolumn{3}{|c|}{ Time series analysis } \\
\hline & 2011 & 2012 & 2013 & 2014 & 2015 & 2016 & Trend & $\beta$ & $P$ value \\
\hline Imipenem & 23.1 & 20.9 & 15.2 & 16.5 & 15.3 & 15.8 & Stable & -1.4811 & 0.1008 \\
\hline Levofloxacin & 28.1 & 28.1 & 20.5 & 10.0 & 10.1 & 8.1 & Decreasing & -4.7833 & 0.0137 \\
\hline Ciprofloxacin & 18.2 & 18.2 & 13.5 & 11.6 & 10.5 & 7.5 & Decreasing & -2.2677 & 0.0011 \\
\hline
\end{tabular}


Table 6 Correlation between antibiotic intensity of consumption and resistance rates of Escherichia coli,Klebsiella pneumoniae, Pseudomonas aeruginosa and incidence rate of methicillin-resistant Staphylococcus aureus

\begin{tabular}{|c|c|c|c|c|c|c|c|c|}
\hline \multirow[b]{2}{*}{ Antibiotics } & \multicolumn{2}{|l|}{ E. coli } & \multicolumn{2}{|c|}{ K. pneumoniae } & \multicolumn{2}{|c|}{ P. aeruginosa } & \multicolumn{2}{|l|}{ MRSA } \\
\hline & $r$ & $P$ value & $r$ & $P$ value & $r$ & $P$ value & $r$ & $P$ value \\
\hline Imipenem/Cilastatin & 0.8651 & 0.0261 & 0.9050 & 0.0131 & -0.7477 & 0.0875 & -0.9611 & 0.0022 \\
\hline Levofloxacin & 0.8954 & 0.0158 & 0.7523 & 0.0844 & 0.8954 & 0.0159 & 0.9450 & 0.0045 \\
\hline Ciprofloxacin & 0.8950 & 0.0160 & 0.9209 & 0.0091 & 0.9282 & 0.0075 & 0.8883 & 0.0180 \\
\hline
\end{tabular}

Antibiotics refer to intensity of consumption (DDD/100 bed-days); bacteria (E. coli, K. pneumoniae and $P$. aeruginosa) refer to their resistance rates (\%) to antibiotics; MRSA refers to incidence rate of MRSA (\%). $r$, correlation coefficient.

of clinical application of antibacterial to control bacteria resistance, which required medical institutions to gather, archive and analyse patient information with respect to the use of carbapenems, to help control the prevalence of CRE. ${ }^{30}$

CHINET surveillance of AMR in China reported the resistance trends from 2005 to 2014, using data from 19 hospitals. $^{31}$ In our hospital, the resistance rates of $E$. coli, $K$. pneumoniae and $P$. aeruginosa to imipenem/cilastatin and meropenem in $2014(0.4 \%$ and $0.9 \%, 4.8 \%$ and $4.1 \%, 16.5 \%$ and $12.9 \%$, respectively) were significantly lower than those reported by CHINET $(0.9 \%$ and $1.0 \%$, $10.5 \%$ and $13.4 \%, 26.6 \%$ and $24.3 \%$, respectively). This proved that AMS in our hospital played an important role in control of AMR.

Some limitations of this study should be noted. First, this was a retrospective observational study without simultaneous control group, the bias could not be well controlled, it was less convincing than a prospective, controlled study design. So the favourable results obtained cannot be attributed solely to the pharmacist intervention, which were affected by many factors. Second, because AMS has been ongoing for many years, several different clinical pharmacists have successively participated in the evaluation of prophylactic antibiotic use; therefore, the evaluation results might be affected slightly by individual differences.

\section{CONCLUSION}

This study demonstrated that AMS in our hospital could reduce and optimise antibiotic use, declining bacterial resistance to FQs was associated with its reduced consumption. Clinical pharmacists played an important role in improving the rational use of antibiotics, however, hospital infection prevention and control measures, national policy guidance all contributed to it. The findings of our study indicate some directions to pursue in controlling the prevalence of CRE and MRSA. AMR is rising worldwide, so continual effort regarding AMS is critical in large hospitals and in primary or community hospitals.
Acknowledgements The authors would like to thank Guosheng Feng and Zhaohui Tong, directors of Beijing Chaoyang Hospital, for supporting and promoting antimicrobial stewardship work. The authors would also like to thank Analisa Avila, ELS, of Liwen Bianji, Edanz Group China (www.liwenbianji.cn/ac), for editing the English text of a draft of this manuscript.

Contributors LHL was the leader of AMS and responsible for supervising the work, work plan and manuscript review. HGW was responsible for carrying out AMS and collecting, analysing and reporting data; she wrote this manuscript and made some amendments after review. HW was responsible for statistical analysis and wrote the analysis method in the manuscript. XJY, HZ, BYL, GC, ZKY, YW, XLC, YYZ, RZ, ZHW, HY were all involved in the different aspects of data collection. PW and CXY provided the bacterial resistance data. All authors were involved in data verification

Funding The authors have not declared a specific grant for this research from any funding agency in the public, commercial or not-for-profit sectors.

Competing interests None declared.

Patient consent for publication Not required.

Ethics approval This study was approved by the Ethics Committee of Beijing Chaoyang Hospital (approval number: 2017-11-28-3).

Provenance and peer review Not commissioned; externally peer reviewed.

Data sharing statement The authors state that if their manuscript is accepted and published, they would be pleased to share the data with readers to improve the rational use of antibiotics.

Open access This is an open access article distributed in accordance with the Creative Commons Attribution Non Commercial (CC BY-NC 4.0) license, which permits others to distribute, remix, adapt, build upon this work non-commercially, and license their derivative works on different terms, provided the original work is properly cited, appropriate credit is given, any changes made indicated, and the use is non-commercial. See: http://creativecommons.org/licenses/by-nc/4.0/.

\section{REFERENCES}

1. Prestinaci F, Pezzotti P, Pantosti A. Antimicrobial resistance: a global multifaceted phenomenon. Pathog Glob Health 2015;109:309-18.

2. Xiao $\mathrm{YH}$, Giske CG, Wei ZQ, et al. Epidemiology and characteristics of antimicrobial resistance in China. Drug Resist Updat 2011;14(45):236-50.

3. World Health Organization. Global strategy for containment of antimicrobial resistance. 2001.

4. National Health and Family Planning Commission of the People's Republic of China. Notice regarding National Special Measure Scheme on Clinical Use of Antibiotics[in Chinese]. 2011 http://www. nhfpc.gov.cn/zwgkzt/wsbysj/201104/51376.shtml.

5. Zhang HX, Li X, Huo HQ, et al. Pharmacist interventions for prophylactic antibiotic use in urological inpatients undergoing clean or clean-contaminated operations in a Chinese hospital. PLoS One 2014;9:e88971.

6. Davey P, Brown E, Charani E, et al. Interventions to improve antibiotic prescribing practices for hospital inpatients. Cochrane Database Syst Rev 2013;4:CD003543.

7. Ma X, Xie J, Yang Y, et al. Antimicrobial stewardship of Chinese ministry of health reduces multidrug-resistant organism isolates 
in critically ill patients: a pre-post study from a single center. $B M C$ Infect Dis 2016:16:704.

8. Xu J, Duan X, Wu H, et al. Surveillance and correlation of antimicrobial usage and resistance of Pseudomonas aeruginosa: a hospital population-based study. PLoS One 2013;8:e78604.

9. Horikoshi Y, Suwa J, Higuchi H, et al. Sustained pediatric antimicrobial stewardship program with consultation to infectious diseases reduced carbapenem resistance and infection-related mortality. Int J Infect Dis 2017;64:69-73.

10. Wushouer $\mathrm{H}$, Zhang ZX, Wang JH, et al. Trends and relationship between antimicrobial resistance and antibiotic use in Xinjiang Uyghur Autonomous Region, China: Based on a 3 year surveillance data, 2014-2016. J Infect Public Health 2018;11:339-46.

11. Cusini A, Herren D, Bütikofer L, et al. Intra-hospital differences in antibiotic use correlate with antimicrobial resistance rate in Escherichia coli and Klebsiella pneumoniae: a retrospective observational study. Antimicrob Resist Infect Control 2018;7:89.

12. Notice Regarding Implementing Stewardship of Antibacterial Use in Clinic in April 2014 [in Chinese]. 2014 http://www.nhfpc.gov. cn/yzygj/s3593/201404/49973560ddde4293a489b158081d777c. shtml.

13. National Health and Family Planning Commission of the People's Republic of China. Regulations on Clinical Applications of Antibiotics [in Chinese]. 2012 http://www.nhfpc.gov.cn/yzygj/s3593/201205/e5e d852b86c4afa8b09a0e58e09e10e.shtml.

14. World Health Organization. Collaborating Center for Drug Statistics Methodology:Guidelines for ATC classification and DDD assignment. 2017 http://wwwwhoccno/filearchive/publications/2017_guidelines_ webpdf.

15. Bao L, Peng R, Wang Y, et al. Significant reduction of antibiotic consumption and patients' costs after an action plan in China, 20102014. PLoS One 2015;10:e0118868.

16. Alawi MM, Darwesh BM. A stepwise introduction of a successful antimicrobial stewardship program. Experience from a tertiary care university hospital in Western, Saudi Arabia. Saudi Med J 2016;37:1350-8.

17. Song P, Li W, Zhou Q. An outpatient antibacterial stewardship intervention during the journey to $\mathrm{JCl}$ accreditation. $\mathrm{BMC}$ Pharmacol Toxicol 2014;15:8.

18. Dinh A, Duran C, Davido B, et al. Impact of an antimicrobial stewardship program to optimize antimicrobial use for outpatients at emergency department. J Hosp Infect 2017:6701:30387.

19. Doernberg SB, Dudas V, Trivedi KK. Implementation of an antimicrobial stewardship program targeting residents with urinary tract infections in three community long-term care facilities: a quasiexperimental study using time-series analysis. Antimicrob Resist Infect Control 2015;4:54.
20. Jenkins TC, Knepper BC, Shihadeh K, et al. Long-term outcomes of an antimicrobial stewardship program implemented in a hospital with low baseline antibiotic use. Infect Control Hosp Epidemiol 2015;36:664-72.

21. Li Z, Cheng B, Zhang K, et al. Pharmacist-driven antimicrobial stewardship in intensive care units in East China: A multicenter prospective cohort study. Am J Infect Control 2017:45:983-9.

22. Bratzler DW, Dellinger EP, Olsen KM, et al. Clinical practice guidelines for antimicrobial prophylaxis in surgery. Am J Health Syst Pharm 2013;70:195-283.

23. Charbonneau P, Parienti JJ, Thibon P, et al. Fluoroquinolone use and methicillin-resistant Staphylococcus aureus isolation rates in hospitalized patients: a quasi experimental study. Clin Infect Dis 2006;42:778-84.

24. Weber SG, Gold HS, Hooper DC, et al. Fluoroquinolones and the risk for methicillin-resistant Staphylococcus aureus in hospitalized patients. Emerg Infect Dis 2003;9:1415-22.

25. Aldeyab MA, Scott MG, Kearney MP, et al. Impact of an enhanced antibiotic stewardship on reducing methicillin-resistant Staphylococcus aureus in primary and secondary healthcare settings. Epidemiol Infect 2014;142:494-500.

26. Lawes T, Edwards B, López-Lozano JM, et al. Trends in Staphylococcus aureus bacteraemia and impacts of infection control practices including universal MRSA admission screening in a hospital in Scotland, 2006-2010: retrospective cohort study and time-series intervention analysis. BMJ Open 2012;2:e000797.

27. Tacconelli E, De Angelis G, Cataldo MA, et al. Does antibiotic exposure increase the risk of methicillin-resistant Staphylococcus aureus (MRSA) isolation? A systematic review and meta-analysis. $J$ Antimicrob Chemother 2008;61:26-38.

28. Borde JP, Kaier K, Steib-Bauert M, et al. Feasibility and impact of an intensified antibiotic stewardship programme targeting cephalosporin and fluoroquinolone use in a tertiary care university medical center. BMC Infect Dis 2014;14:201.

29. Lai CC, Chu CC, Cheng A, et al. Correlation between antimicrobial consumption and incidence of health-care-associated infections due to methicillin-resistant Staphylococcus aureus and vancomycinresistant enterococci at a university hospital in Taiwan from 2000 to 2010. J Microbiol Immunol Infect 2015;48:431-6.

30. National Health and Family Planning Commission (NHFPC) of the People's Republic of China. Notice regarding Further reinforcement the Management of Clinical Application of Antibacterial to Control Bacteria Resistant [in Chinese]. 2017 http://www.nhfpc.gov.cn/yzygj/ s7659/201703/d2f580480cef4ab1b976542b550f36cf.shtml.

31. Hu FP, Guo Y, Zhu DM, et al. Resistance trends among clinical isolates in China reported from CHINET surveillance of bacterial resistance, 2005-2014. Clin Microbiol Infect 2016;22(Suppl 1):S9-14. 